\title{
Direct Simulation of a Turbulent Oscillating Boundary Layer
}

Philippe R. Spalart and Barrett S. Baldwin

(NASA-Ty-89460) DIRECT SILOTEIICA OF A TURBULENT OSCILLATING ECUYCAEY LAYEB

9 F Avil: NIIS EC BO2/UF DO CSCL 20D
N87-24642

$\begin{array}{ll}\text { C3 } & \text { Unclas } \\ & 0080332\end{array}$

May 1987 
NASA Technical Memorandum 89460

\section{Direct Simulation of a Turbulent Oscillating Boundary Layer}

Philippe R. Spalart,

Barrett S. Baldwin, Ames Research Center, Moffett Field, California

May 1987

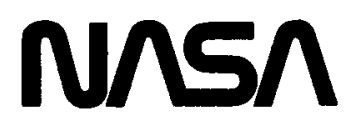


DIRECT SIMULATION OF A TURBULENT OSCILLATING BOUNDARY LAYER

Philippe R. SPALART and Barrett S. BALDWIN

NASA Ames Research Center

\begin{abstract}
The turbulent boundary layer under a freestream velocity that varies sinusoidally in time around a zero mean is considered. The flow has a rich variety of behaviors including strong pressure gradients. inflection points, and reversa. A theory for the velocityand stress profiles at high Reynolds number is formulated. Wellresolved direct Navier-Stokes simulations are conducted over a narrow range of Reynolds numbers and the results are compared with the theoretical predictions. The flow is also computed over - wider range of Reynolds numbers using a new algebraic turbulence model: the results are compared with the direct simulations and the theory.
\end{abstract}

\section{NOMENCLATURE}

$f_{1}, f_{2} \ldots \quad$ unknown nondimensional functions, see Eq. (3), (4)... $L \equiv r_{0} / \omega$ outer length scale

Re - $l_{0} \delta_{l} / \nu$ Reynolds number

$t$ time

$u, u, u$ velocity components

$u^{\prime}=u \quad l$ fluctuating volocity component

$l^{\prime} \equiv\langle u\rangle$ mean velocity component

$U_{l}$ laminar mean velocity component, see Eq. (3)

$U_{0}$ peak freestream velocity, see Eq. (1)

$U_{\infty} \quad$ freestream velocity, see Eq. (1)

$u^{*} \quad$ peak friction velocity, see Eq. (5)

$u_{r}$ friction velocity (can be negative)

$U^{+} \equiv U / u_{r} \quad$ wall units

$x, y, z$ streamwise, normal, spanwise coordinates

$y^{+} \boxminus y\left|u_{+}\right| / \nu$ wall units

$\delta_{1} \equiv \sqrt{2 \nu / \omega}$. laminar boundary-layer thickness, see Eq. (3)

$\delta$ turbulent boundary-layer thickness, see Eq. (5)

* Karman constant

$\Lambda_{z}, \Lambda_{z}$ periods in $x$ and $z$ directions

$\nu$ kinematic viscosity

$\phi \equiv \omega t$ phase angle

$\phi$ phase shift, a function of $R e$, see Eq. (7)

$\omega$ frequency, see Eq. (1)

$r \equiv U_{y}-\left\langle u^{\prime} v^{\prime}\right\rangle \quad$ total shear stress

$<>$ average in $x, z$ and/or ensemble

\section{INTRODUCTION}

The flow under consideration is the turbulent version of a classical exact solution of the Navier-Stokes equations, known as Stokes' second problem (1). The domain is the half-space over a stationary flat plate and the freestream velocity is given by

$$
U_{\infty}(t)=U_{0} \cos (\omega t)
$$

The three parameters (peak veloriny $l_{0}$, frequency $\omega$ and kinematir viscosity $\nu$ ) combine into a single nondimensional parameter: a Reynolds number Re. The laminar solution is

$$
U_{l}(y, t)=U_{\infty}(t)-U_{0} e^{-y / \delta_{1}} \cos \left(\omega t-y / \delta_{l}\right)
$$

where $\delta_{l}$ is the laminar boundary-layer thickness.

This flow is attractive because of its rich time-dependent behavior, and because it is naturally homogeneous in the directions parallel to the plate (this is true only because the mean value of $U_{\infty}(t)$ is zero). This justifies imposing periodic conditions in a direct simulation and considerably improves the statistical sample. In addition, the Reynolds-averaged quantities are functions only of the normal coordinate and the time. This provides a good test case for turbulence models, in which their ability to treat unsteady and inflectional velocity profiles can be tested with little numerical effort and with high numerical accuracy. It also exhibits reversal of the velocity without the usual viscous-inviscid coupling diffculties and singularities encountered in spatially-separating flows. The fully-developed flow will most likely be close to the common turbulent boundary layer, except that the lack of entrainment will prevent the formation of a sharp laminar-turbulent interface.

The known results concern primarily the transition Reynolds number, $R_{\epsilon} \approx 600$, and the stability of the laminar solution to small disturbances (2). Detailed experimental results are not available for validation. However the only source of error is numerical: an approximate treatment of the boundary-layer growth is not needed here as it was in Ref. 3. The numerical errors can be reliably estimated by conducting simulations with improved resolution, enlarged domains, and so on, and by using the experience gained while simulating other flows $(3,4)$. The agreement with well-established laws (e. g., the log law) also helps build confidence in the numerical results.

\section{TENTATIVE THEORY OF THE FLOW}

The theory is tentative in that it is based on assumptions which are plausible and consistent with current thinking, but cannot be rigorously proven. As is often the case in turbulence, only neces. sary conditions will be obtained. Also, the evidence supporting the theory is encouraging, but still limited. We focus on the mean velocity $U[y, t]$ and the total shear stress $r[y, t]$. They are linked by the moment um equation, $U_{t}-U_{\infty t}=\tau_{y}$. For clarity we use the following notation: square brackets surround the arguments of a function, while parentheses group terms in an expression. Most of the derivation can be generalized to other laws than cosiw $t$ for $U_{\infty} / U_{0}$. 
Define the phase $\phi$ and the length seale $L$. Dimensional analysis shows that $U$ and $T$ must have the following form:

$$
\begin{gathered}
U[y, t]-U_{\infty}[t]=U_{0} f_{1}\left[\frac{y}{L}, \phi, R e\right] \\
\tau[y, t]=U_{0}^{2} f_{2}\left[\frac{y}{L}, \phi, R e\right]
\end{gathered}
$$

for some nondimensional functions $f_{1}$ and $f_{2}$. Recall that the averages are taken over $x$ and $z$ with sufficiently large domains and by phase-averaging. The momentum equation and boundary condition become

$$
\frac{\partial f_{1}}{\partial \phi}=\frac{\partial f_{2}}{\partial(y / L)}, \quad f_{1}[0, \phi, R e]=-\frac{U_{\infty}}{U_{0}}[\phi] .
$$

So far no appraximations have been made. The functions $f_{1}$ and $f_{2}$ are functions of three variables; the objective is to separate the dependence on $R e$ from the other two as much as possible.

\section{Quter region}

The Reynolds-number-similarity hypothesis (5) asserts that, away from the wall, the gross features of the turbulence (at least $U$ and $\tau$, which do not depend strongly on the small-scale motion) can be independent of the Reynolds number provided that proper velocity- and length scales $u^{*}$ and $\delta$ (different from $U_{0}$ and $L$ ) are chosen to normalize them. In the outer region we replace Eq. (3) by

$$
\begin{gathered}
U[y, t]-U_{\infty}[t]=u^{\bullet} f_{3}\left[\frac{y}{\delta}, \phi, R e\right], \\
\tau[y, t]=u^{* 2} f_{4}\left[\frac{y}{\delta}, \phi, R e\right] .
\end{gathered}
$$

This equation is just as general as Eq. (3), since $u^{*} / U_{0}$ and $\delta / L$ are functions of Re, but $f_{3}$ and $f_{4}$ are now of order 1 , as is the range of $y / \delta$ over which most of their variation occurs. We can define $u^{*}$ as $\sqrt{\tau_{\max }}$ where $\tau_{\max }$ is the peak value of the shear stress at the wall during the cycle. For $\delta$ there is no such obvious, quantitative definition (especially since the flow does not have a sharp laminar-turbulent interface). Since the time scale is $1 / \omega$. $\delta$ must be proportional to $u^{*} / \omega$. We can use this to define $\delta$. We are free to choose the value of the ratio, and we choose 1 . Therefore:

$$
\delta \equiv \frac{u^{*}}{\omega} .
$$

Thus $\delta$ is proportional to the thickness of the turbulent region, but not equal to it. Because of Eq. (6), $f_{3}$ and $f_{4}$ satisfy an equation analogous to Eq. (4(a)), simply replacing $L$ with $\delta$.

We now introduce the major assumption: the only dependence of $f_{3}$ and $f_{4}$ on $R e$ is a phase shift $\left.\phi_{0} \mid R e\right]$ (as we shall see, the theory is too limited if we assume that $f_{3}$ and $f_{4}$ do not depend on $R e$ at all). The idea behind this assumption is that at different Reynolds numbers $U-U_{\infty}$ and $\tau$ (scaled with $u^{*}$ and $\delta$ ) may have the same behavior, but not at exactly the same phase relative to $U_{\infty}$. The assumption is expressed as follows:

$$
\begin{aligned}
& f_{3}\left[\frac{y}{\delta}, \phi, R e\right]=f_{3}\left[\frac{y}{\delta}, \phi+\phi_{0}[R e]\right], \\
& f_{4}\left[\frac{y}{\delta}, \phi, R_{e}\right]=f_{6}\left[\frac{y}{\delta}, \phi+\phi_{0}[R e]\right] .
\end{aligned}
$$

Note that $f_{s}$ and $f_{0}$ are functions of only two variables. On the other hand we have introduced three new functions of $R_{e}: u^{*} / U_{0}$, $\delta / L$ and $\phi_{0}$ (but recall that the first two are related by Eq. (6)).

\section{Inner rerion}

Bere we apply the usual arguments. The variation of $r$ with $y$ is neglected, so $\tau[y, t] \approx \tau[0, t]$, and the momentum equation is not considered. Define $u_{+} \equiv \sqrt{\tau[0, t]}$ where the square root is taken to have the sign of the argument. The velocity $U$ is assumed to follow the law of the wall:

$$
U=u_{r} f_{7}\left[\frac{y\left|u_{\tau}\right|}{\nu}\right]
$$

Clearly when the wall shear stress reverses $(\tau[0, t]$ vanishes). Ilius ansumption breaks down. The theory will nor apply near the phase of reversal, but this may not have much effect on the rest of the cycle.

\section{Qverlap argument}

In the overlap region both Eq. (7a) and Eq. (8) are assumed to be valid and we obtain

$$
\frac{U_{0}}{u^{*}} \frac{U_{\infty}}{U_{0}}[\phi]+f_{3}\left[\frac{y}{\delta}, \phi+\phi_{0}\right]=\frac{u_{\tau}}{u^{*}}\left[\phi+\phi_{0}\right] f_{7}\left[\frac{y}{\delta} \frac{\left|u_{\tau}\right|}{u^{*}} \frac{u^{*} \delta}{\nu}\right]
$$

To exploit the overlap argument we can, for instance, vary $R e$ in Eq. (9) while holding $y / \delta$ and $\phi+\phi_{0}$ fixed. We obtain

$\frac{U_{\infty}}{U_{0}} \frac{d}{d R e}\left(\frac{U_{0}}{u^{*}}\right)-\frac{U_{0}}{u^{*}} \frac{d}{d \phi}\left(\frac{U_{\infty}}{U_{0}}\right) \frac{d \phi_{0}}{d R e}=\frac{u_{\tau}}{u^{*}} \frac{d f_{\tau}}{d\left(\log \left[y^{+}\right]\right)} \frac{d\left(\log \left[u^{*} \delta / \nu\right]\right)}{d R e}$

The only term that depends on $y$ is the last one, which contains $d f_{7} / d\left(\log \left(y^{+} \mid\right)\right.$. Therefore this quantity must be a constant, e.g., $1 / \kappa$; again the overlap argument predicts a log layer $\left(f_{T}=\log \left[y^{+}\right] / \kappa+C\right)$.

The reason $\phi_{0}(R e)$ was introduced is not apparent. Consider the variation of the various terms in Eq. (10) as a function of $\phi$. If $\phi_{0}$ were independent of $R e$, the second term would be 0 . Then during the cycle, $u_{r} / u^{*}$ (the third term) would be proportional to $U_{\infty} / U_{0}$ (first term), so that the wall shear stress would have to have the same phase as the freestream velocity, which is physically not correct. The variable- $\phi_{0}$ model allows the deceleration to enter through $d\left(U_{\infty} / U_{0}\right) / d \phi$ (second term) and make the wall stress "lead" the freestream velocity and reverse earlier (if $d \phi_{0} / d R e<0$ ).

\section{Dependence on $\phi$ and $\phi$ n}

We conclude the analysis by exploiting the dependence of Eq. (10) on $\phi$ and $\phi_{0}$. Using Eq. (1) we see that the left-hand-side is proportional to $\cos \left(\phi+\phi_{1}\right)$ with $\phi_{1}$ defined by $\left.\tan \mid \phi_{1}\right] \equiv d \phi_{0} / d\left(\log \left[u^{*} / U_{0}\right]\right)$. The right-hand-side is proportional to $u_{r} / u^{*}$ which is a function of $\phi+\phi_{0}$. Therefore $\phi_{1}-\phi_{0}$ is a constant. Since $\phi_{0}$ is meaningful only up to an additive constant (see Eq. (7)), we can choose the constant so that $\phi_{0}=\phi_{1}$. Consequently $\tan \left[\phi_{0}\right]=d \phi_{0} / d\left(\log \left[u^{*} / U_{0}\right]\right)$, which integrates to

$$
\sin \left[\phi_{0}\right]=A \frac{u^{*}}{U_{0}}
$$

where $A$ is a constant. Also, $u^{*}$ was defined so that the peak value of $u_{\tau} / u^{*}$ is 1 . Therefore

$$
\frac{u_{r}}{u^{*}}=\cos \left[\phi+\phi_{0}\right]
$$

Finally, rearranging Eq. (10) and using Eq. (6) to eliminate $\delta$,

$$
-\left(\frac{A \kappa}{2 \sin \left[\phi_{0}\right]}+\cos \left[\phi_{0} \mid\right) \frac{d \phi_{0}}{\sin \left[\phi_{0}\right]}=\frac{d R e}{R e}\right. \text {. }
$$

This integrates to

$$
\frac{\kappa}{2} \frac{U_{0}}{u^{*}} \cos \left[\phi_{0}\right]+\log \left[\frac{U_{0}}{u^{*}}\right]=\log [R e]-B
$$

where $B$ is a constant. It turns out that $\phi_{0}$ is small, of the order of 0.15 radians, so that $\cos \left|\phi_{0}\right| \approx 0.99$. If it is replaced by 1 , Eq. (14) involves only $u^{*} / U_{0}$ and Re.

Equations (11), (12), and (14) provide strong predictions that can be checked against sufficiently accurate simulations. Such a check will also provide the values of $A$ and $B$. The theory predicts that as $R e \rightarrow \infty, u^{*} / U_{0}, \delta / L$, and $\phi_{0}$ all tend to 0 , although slowly (roughly like $1 / \log (R e)$, as in other flows). Also note that the nondimensional shear-stress gradient $d \tau^{+} / d y^{+}$is of order $\left(U_{0} / u^{*}\right)^{2} / R e^{2}$; it also tends to zero as $R e \rightarrow \infty$ so that the "almost-constant-stress" assumption for the inner region becomes more and more ralid. 
The known limitations of the theory are that it applies only at sufficiently high Reynolds number and that it does not apply near the phase of reversal of the wall shear stress. This failure near reversal is obvious since Eq. (12) predicts that $\tau$ has a double zero when it reverses, which is counterintuitive and is not indicated by the simulation results. It may be possible to extend the theory to cover the reversal region by matching asymptotic expansions, but this has not yet been achieved.

\section{DIRECT-SIMULATION RESULTS}

The numerical method used to solve the time-dependent, threedimensional, incompressible Navier-Stokes equations is described in detail in Ref. 6. It is fully spectral in space and second-orderaccurate in time. The method was further tested by solving the linear stability equations for the laminar solution using Floquet theory (in that case the time integration used a fourth-orderaccurate, implicit, reversible scheme). The results obtained by Hall (7) with a very different method were reproduced, which shows that the spectral basis functions that are used are well adapted to the flow. The results indicate linear stability at all of the Reynolds numbers and wave numbers that were investigated (up to $R e=2000$ ). For the turbulent simuiations, the boundary condition (Eq. 1) is most simply enforced by solving for the difference between the flow and the laminar flow $U_{1}$ : this difference satisfies homogeneous boundary conditions at $y=0$ and $y \rightarrow \infty$.

The periods are $\Lambda_{z}=0.12 L$ and $\Lambda_{z}=0.06 L$ (about $60 \delta_{l}$ and $\left.30 \delta_{l}\right)$. The resolution is as follows. In the $y$-direction there are 10 collocation points within about 10 wall units of the wall. In the $x$ - and $z$-directions the spacing is about 20 and 7 wall units, respectively. Random numbers are used as initial disturbances in order to break any symmetries in the flow field. Their amplitude has to be large enough; otherwise the flow remains laminar (recall that it is linearly stable). The initial transient takes about one cycle and is not included in the phase averages. The parity under the transformation $\phi \rightarrow \phi+\pi$ is used to double the sample (averages are of course taken over $x$ and $z$ as well).

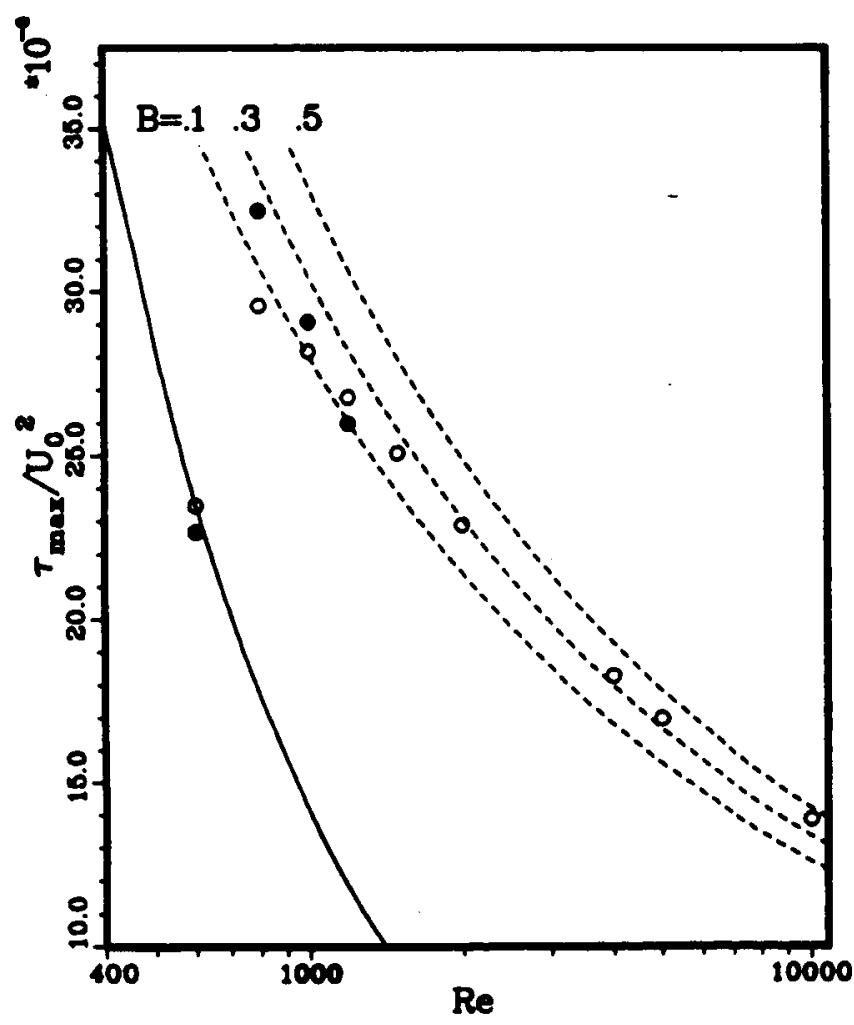

Fig. 1. Peak wall shear streas vs. Reynolds number. - laminar flow; - . - Eq. (14); • direct simulations; 0 turbulence model.
Figure 1 show's $T_{\text {maz }} / C_{0}^{2}$ as a function of Re. The laminar solution and the curves corresponding wo several values of $B$ in Eq. (14) are shown. Direct simulations were innducted at $R_{e}=600,800,1000$, and 1200 . The trend from 800 to 1200 is steeper than predicted by the theory. The range of Reynolds number is too narrow for one to conclude whether the theory is invalidated by the results, or whether the disagreement is simply a low-Reynolds-number effect. The results obtained with the turbulence model are included in the figure, but will be discussed later.
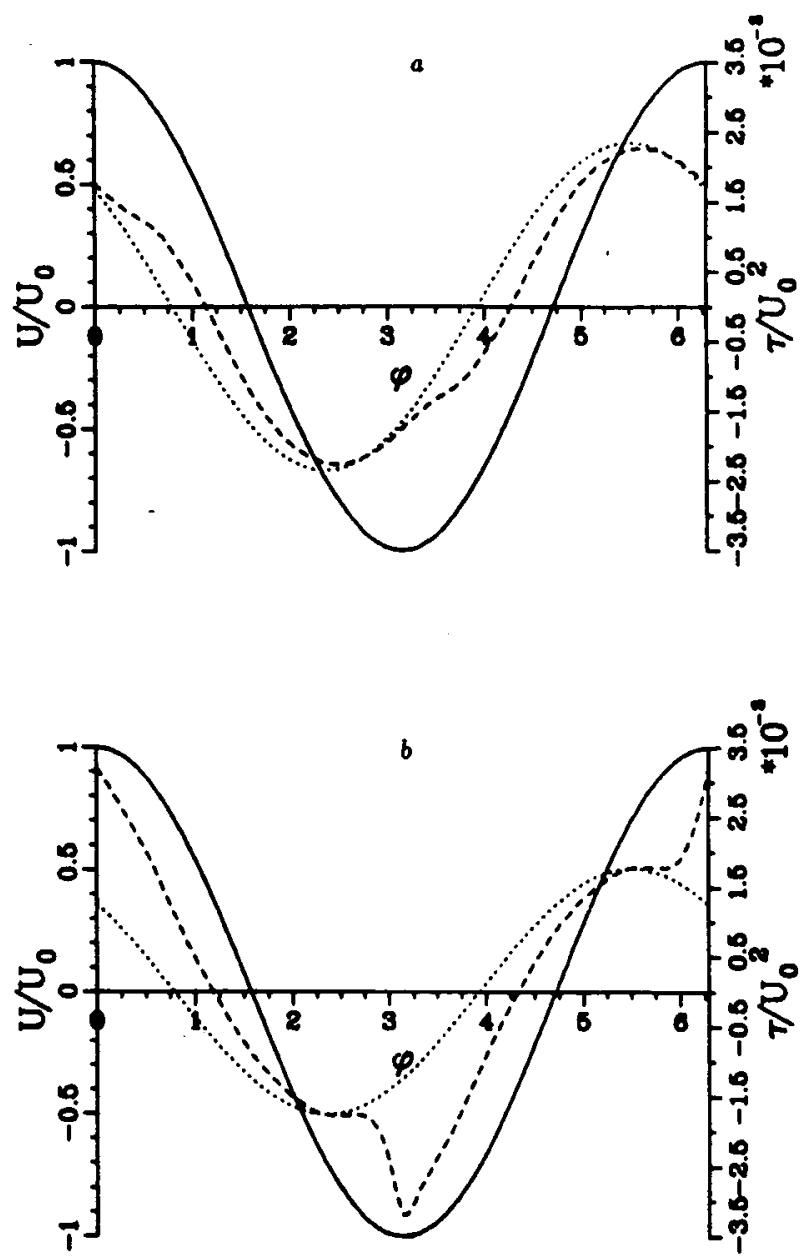

Fig. 2. Freestream velocity and wall shear stress vs. phase angle. $-U_{\infty} / U_{0} ; \cdots+$ in laminar flow; $\cdots T$ in turbulent flow. a) $R e=600$; b) $R e=800$.

At $R e=600$, the peak wall stress is close to the laminar value, but the flow is not laminar. Figure 2(a) compares the turbulent wall stress to the laminar solution; they differ significantly during the deceleration phase. Reversal occurs much later in the turbulent flow. The average power consumption per unit area, normalized by $U_{0}^{3}$, is about $0.98 \times 10^{-3}$ in the turbulent flow compared with $0.83 \times 10^{-3}$ in the laminar flow. The flow is cleariy not laminar, but is also far from being well-developed turbulence. The velocity profile never displays a log layer and the anisotropy of the Reynolds-stress tensor is abnormal. Monkewitz and Bunster $(8)$ visually observed finite-amplitude disturbances, also near the phase of reversal, starting between $R e-554$ and 647 .

Between Reynolds number 800 and 800 a second transition occurs. Figure 2(b) shows that the wall stress is now high dur. 
ing the high-freestream-velocity phase. It now has a sharp peak with a value much larger than in the laminar flow, and the power comsumption is $86 \%$ larger than in laninar flow. Strong turbulence develops around $\phi=2.8$; a log layer forms in the velocity profile near $\phi=0$ and is maintained well into the deceleration phase, around $\phi=0 . \bar{t}$. Reversal now occurs near $\phi=1.25$. The results at $R e=1000$ are similar, but the peak in the wall stress is much wider. Turbulence during the peak-velocity phase was observed in experiments by Merkli and Thomann (9) but their Reynolds number was much lower, about 300 . See also Hino, Sawamoto and Takasu's experiments is a pipe (10).

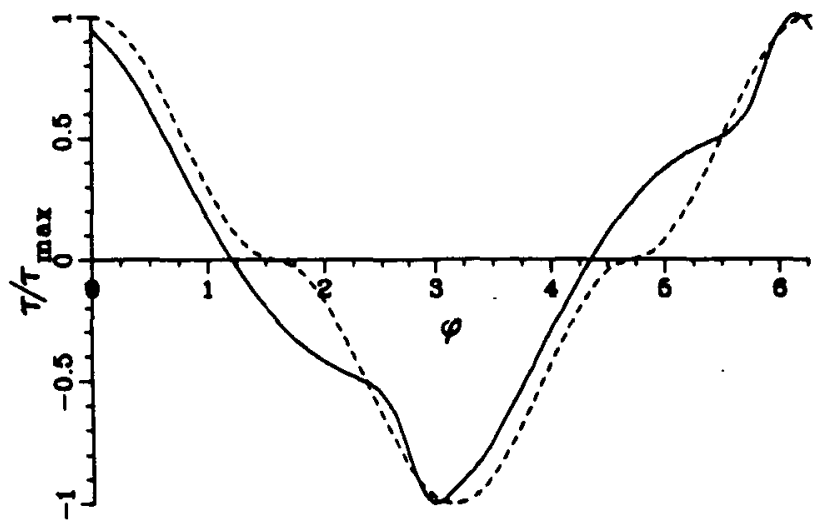

Fig. 3. Wall shear stress vs. phase angle. $R e=1000$. $-\tau / \tau_{\max } ; \cdots \cos (\phi)|\cos (\phi)|$.

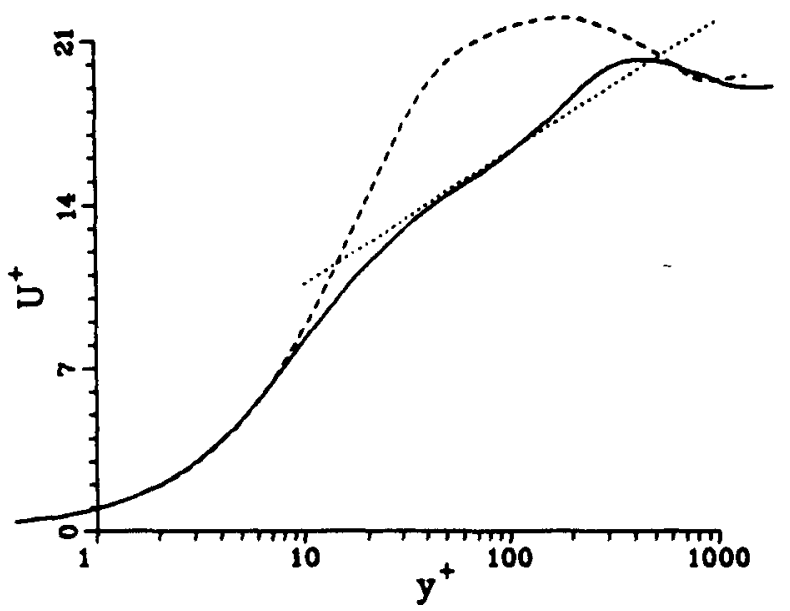

Fig. 4. Mean velocity vs. distance from the wall. $R e=1000$. $-\phi=0 ; \cdots \phi=2.44 ; \cdots \log \left[y^{+}\right] / 0.41+5$.

The agreement with $\mathrm{Eq} .(12)$ is tested in Fig. 3. One expects $\tau / \tau_{\max }$ to have the same shape as $\cos (\phi)|\cos (\phi)|$, only shifted to the left. This is observed only for $\phi$ between about -0.3 and 1 . This is also the range of phases in which a log layer is observed. Figure 4 shows a $\log$ layer at $\phi=0$, but not at $\phi=2.44$. The profile at $\phi=2.44$ is laminar-like: $U^{+}\left[y^{+}\right]$is far above the usual log layer. During the acceleration phase, the favorable pressure gradient delays the development of the turbulence considerably. Transition does not occur until the pressure gradient falls below about 0.02 , in wall units based on the current wall shear stress, even though the near-wall layer is subjected to strong disturbances from the turbulence remaining farther from the wall. Thus one can attribute the lack of agreement with Eq. (12) during that phase to low. Revnolds-number effects. This leck of agreement and the narrow range of Reynolds numbers tnake an accurate check of Eq. $1111 \mathrm{im}$ mossible: one can just note that the value of $A$ is of the order of 3 .
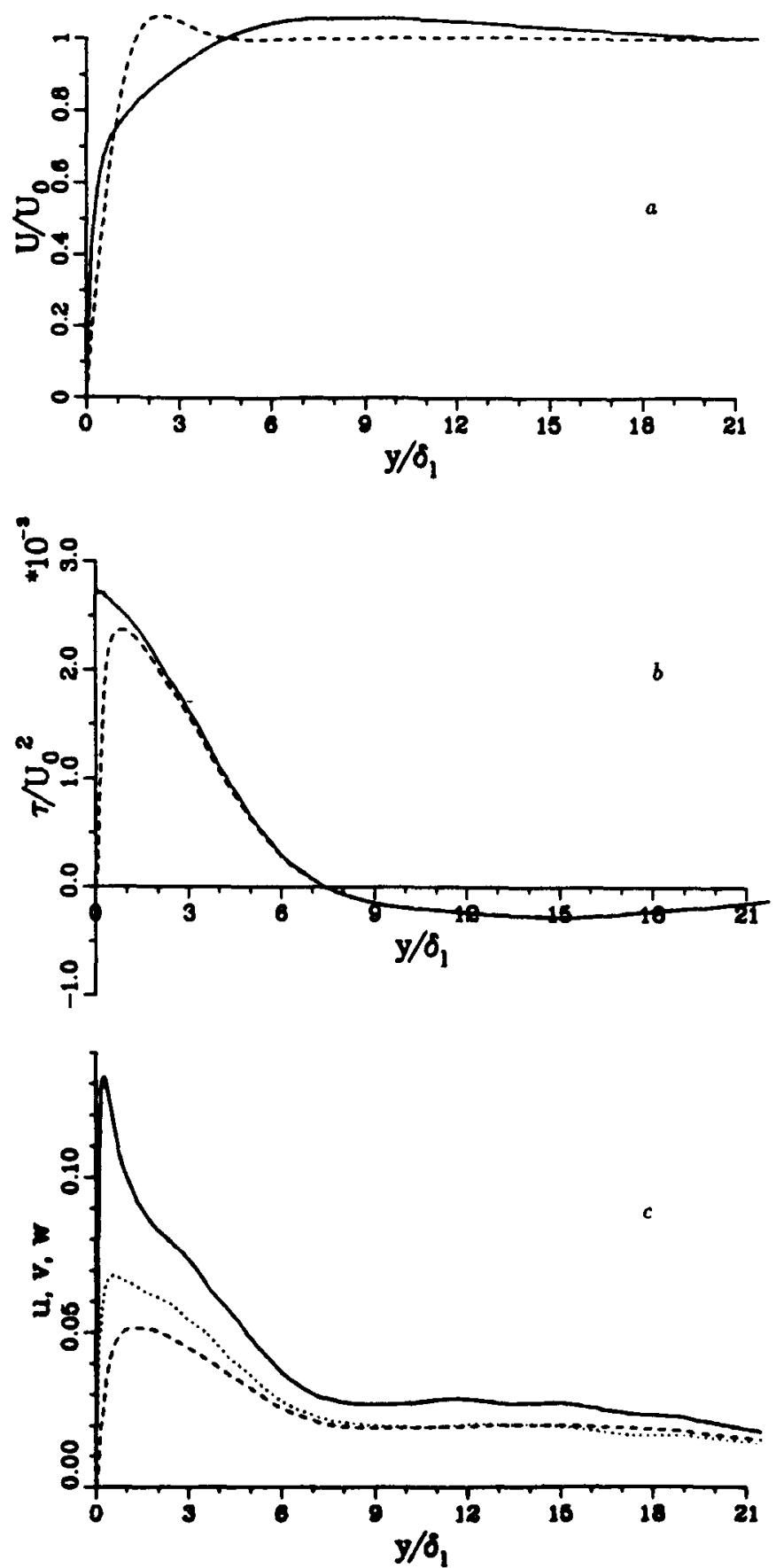

Fig. 5. Profiles in $R e=1000$ flow, $\phi=0$. a) Velocity $U / U_{0} ;-$ turbulent, - - laminar. b) Shear stress $T / U_{0}^{2} ;-$ total stress, -.. Reynolds stress. c) turbulence intensities $u^{\prime} / U_{0}$, etc.: $-u^{\prime}$, $\cdots v^{\prime}, \cdots w^{\prime}$.

Other aspects of the flow will now be described using the $R e=1000$ case, for which the best sample (four half-cycles) happens to be available. Figure 5 shows at $\phi=0$ the mean velocity, the shear stress, and the turbulence intensity in each component. The near-wall behavior is very similar to that of the usual boundary layers, but away from the wall the shear stress crosses 0 and returns to it only slowly, and the intensities have very long "tails". They extend far heyond the region with significant mean shear $U_{y}$. This is caused bv the lack of entgainment. Notice also how a local 
minimum in the turbulence intensity, a zero crossing of the shear stress, and a zero crossing of the mean shear $V_{y}$ all coincide near $y_{1} c_{1}=8$. As a rule, the zero crossings of the stress and the shear tend to follow each other. Since they imply that the turbulentenergy production is 0 , they explain the minimum in the energy.

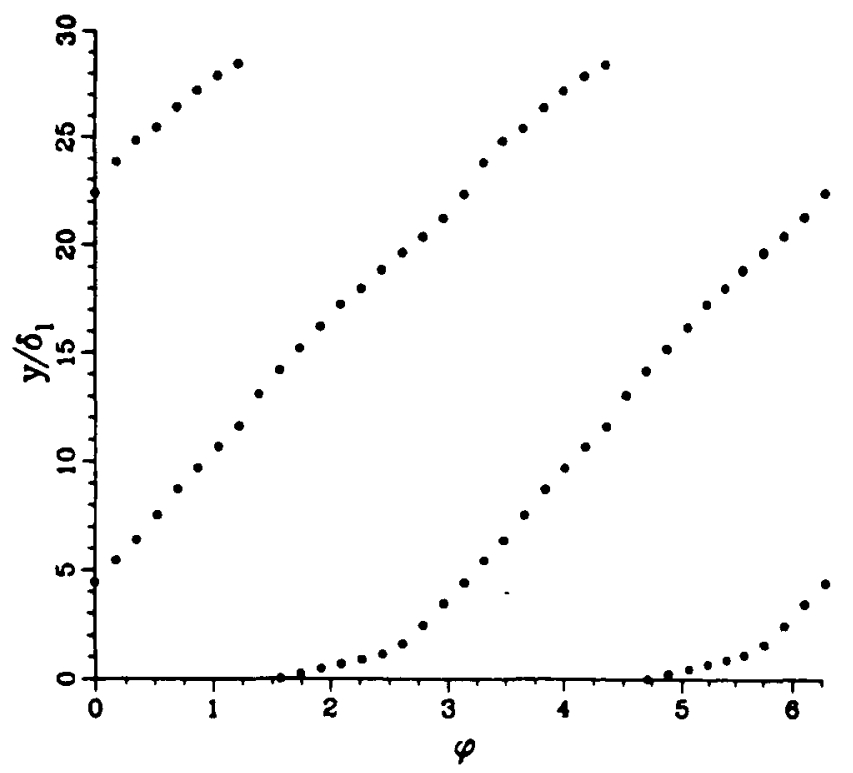

Fig. 6. Zero crossings of $U-U_{\infty}$ vs. phase angle. $R e=1000$.

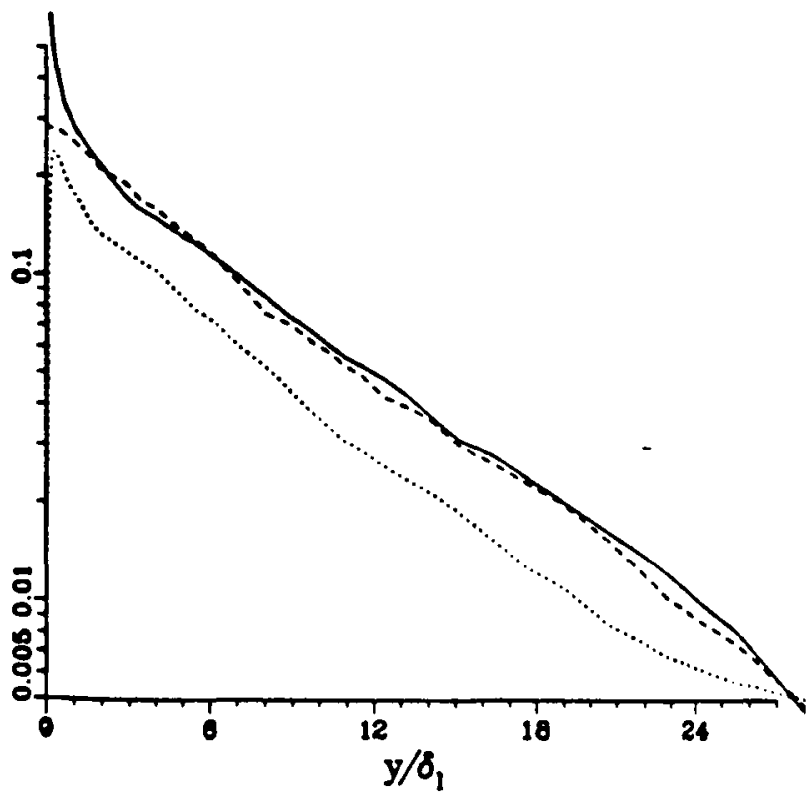

Fig. 7. Peak value of various quantities over a cycle, vs. $y$. $R e=1000 .-\left(U-U_{\infty}\right) ; \cdots 100 \times \tau ; \cdots 10 \times\left(u^{\prime 2}+v^{\prime 2}+w^{\prime 2}\right)$. Normalized by $U_{0}$.

The results further show that the structure of the turbulence (for instance the anisotropy of the Reynolds-stress tensor) at different values of $y / \delta$ is quite similar. The zero crossings of the stress $\tau$, of the mean shear $U_{y}$, and of the deviation from freestream $U-U_{\infty}$ all propagate away from the wall at roughly constant velocity, except close to the wall. Figure 6 shows that the $U=U_{\infty}$ point starts from the wall at $\phi=\pi / 2$ with a slope of 1 (the laminar value), and that near $\phi=2.5$ its velocity abruptly increases to about 6 . The peak values of $\tau$, the turbulent energy, and $U-\nabla_{\infty}$ over a cycle also decay exponentially as functions of $y / \delta$ (Fig. 7). The decay rate is about $T$ times smaller than in laminar flow. Thus there is a strong analogy with the laminar
How (Eq. 2): the "eddy viscosity" would be uniform at about 40 tines the molecular viscosity (at $R e=1000$ ).

These findings were somewhat surprising. It was expected that the propagation velocity of the waves would scale with the local velocity scale, and therefore decrease rapidly away from the wall. For the peak values of $U-U_{\infty}$ and $\tau$, an algebraic decay seemed more likely. Note that a constant viscosity scale and an exponential decay of the velocity scale imply an exponential growth of the length scale. On the other hand, it was found that in the Reynolds-stress budgets, the role of the various terms did change with $y$ : near the wall the usual terms dominate but axay from it, the turbulent-diffusion term becomes much stronger. Thus the similarity is not complete. Even so, the behavior in Figs. 6 and $i$ ought to be explained.

To further document the behavior of the turbulence the $k-\epsilon$ model was applied to the flow. The high-Reynolds number version of the model was used with standard values for the constants $\left(C_{\mu}=0.09, C_{t 1}=1.45, C_{t 2}=1.9, \sigma_{k}=1, \sigma_{t}=1.3\right)(\underline{11})$. Only the region $y>2 \delta_{l}$ was considered: boundary conditions for $U, k$ and $\epsilon$ at $y=2 \delta_{1}$ were taken from the direct simulations $(R e=$ $1000)$. Thus near-wall corrections were not needed. For large $y$ trivial conditions were used $\left(U=U_{\infty}, k=\epsilon=0\right)$. The $k-\epsilon$ results were in very good agreement with the direct simulations, including the uniformity of the eddy viscosity. The agreement was further improved by a slight adjustment of $C_{\mu}$, to 0.085 . The directsimulation results also suggested lower values for $\sigma_{k}$, around 0.8 , but such values did not alter the results noticeably. This exercise shows that, excluding the wall region, the oscillating boundary layer is within the reach of the high-Reynolds-number $k-t$ model. Thus, near-wall corrections to the model could be tested with confidence that the outer region is treated properiy.

\section{ALGEBRAIC-TURBULENCE-MODEL RESULTS}

The eddy-viscosity model used in this paper is algebraic in the sense that the eddy viscosity in a given profile is a specified function. However, one of the parameters depends on the phase angle (in other problems it could depend on time or on the streamwise coordinate) and is determined from solution of an ordinary differential equation. The development of the model follows the procedure originated by Johnson and King (12), but differs in detail. The structure parameter is used to convert the turbulent-energy equation to an equation for $\tau$, which is integrated over the profile to obtain an ordinary differential equation. Models of this type can exhibit transition and relaminarization properties in contrast to conventional algebraic models. A complete description will be given elsewhere.

The peak wall stress obtained with the model between $R e=800$ and 10,000 was shown in Fig. 1. Where the direct-simulation and turbulence-model results overlap, the agreement is acceptable. In that range both sets of results disagree with $\mathrm{Eq}$. (14) since they do not follow a $B=$ constant curve. When $R e$ exceeds about 4,000 , the agreement between the model and Eq. (14) is seen to improve. This suggests that the discrepancy for Re of the order of 1000 is indeed due to low-Reynolds-number effects, for which the theory is not expected to account. The value of $B$ would then be between 0.3 and 0.5 . On the other hand the Reynolds-number-similarity hypothesis and the law-of-the-wall hypothesis, which are central to the theory, are also "built into" the turbulence model. In that sense, the model does not provide a truly independent check of the theory. It is still very useful in assessing the low-Reynoldsnumber effects, which the direct simulations will be unable to do in the near future because of their excessive cost. Note also that the model predicted relaminarization, between $R e=800$ and 600 . 


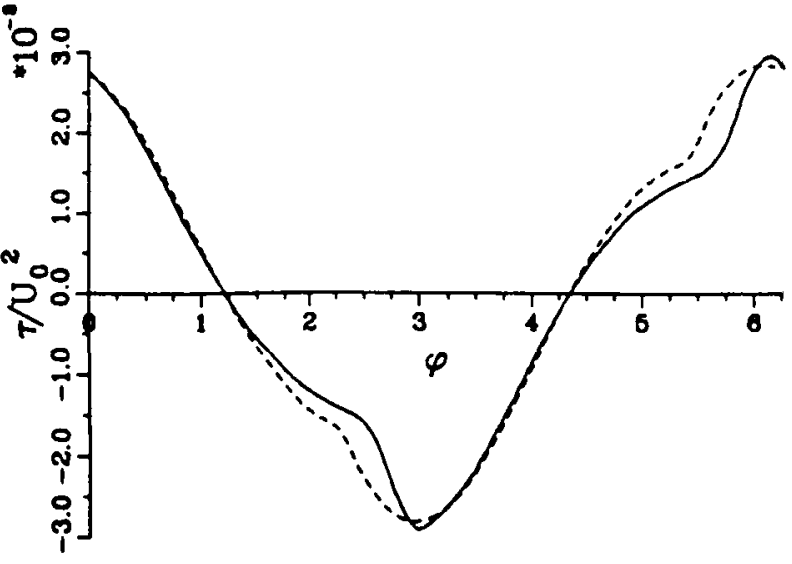

Fig. 8. Wall shear stress vs. phase angle. $R e=1000$. - direct simulation; - - turbulence model.

Figure 8 compares the evolution of the wall stress at $R_{e}=1000$, computed by direct simulation and with the model. The agreement is very good; the model even predicts the quiescent phase around $\phi=2$ and the surge after $\phi=2.5$ (although the surge occurs slightly earlier with the model). The agreement a way from the wall (velocity profiles, etc.) is also good. Finally, Fig. 9 shows $\tau / \tau_{\max }$ versus $\phi$ at $R e=10,000$. The curve follows Eq. (12) well for a much longer part of the cycle than at $R e=1000$ (roughly, from $\phi=-1$ to $\phi=1$ ). The phase shift $\phi_{0}$ is also smaller. The predictions of the model are quite consistent with the theory.

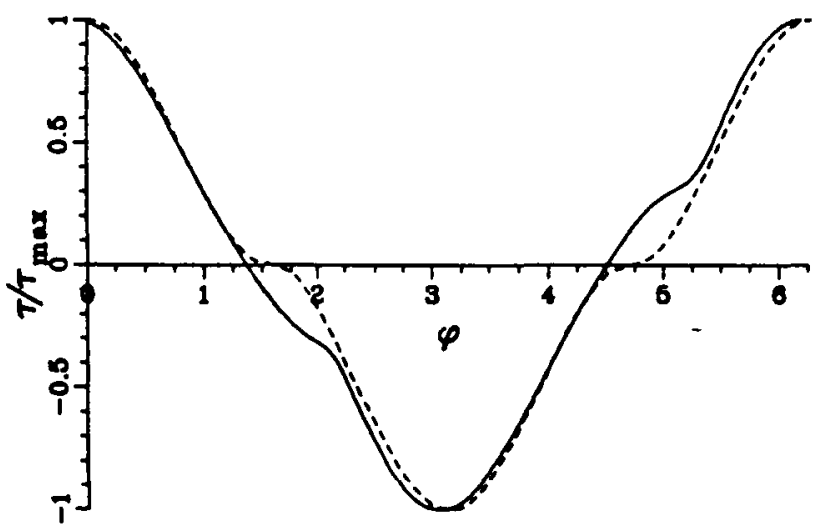

Fig. 9. Wall shear stress vs. phase angle. $R e=10,000$. $-\tau / \tau_{\max } ; \cdots \cos (\phi)|\cos (\phi)|$.

\section{CONCLUSIONS}

The oscillating boundary layer displays a complex behavior, both as a function of phase angle and of Reynolds number. Although it is believed to be linearly stable, it exhibits a first transition to a "pre-turbulent" state just below a Reynolds number of 600 . A second transition, between 600 and 800 , allows it to generate well-developed turbulence during at least part of the cycle. During that part of the cycle, it contains a log layer and agrees with other aspects of a high-Reynolds-number theory that was also presented. Thus the theory is confirmed to some extent by the direct-simulation results. These results even suggest that the theory could be simplified further, which does not seem to be justified by traditional arguments but is also supported by predictions of the $k-\epsilon$ model. A new algebraic turbulence model, deaigned and calibrated on the present flow, yielded satisfactory agreement with the direct simulations and with the theory in spite of the complexity of the flow.
Acknowledgments The first author benefited from discussions with Prof. N. Rott of Stanford University and Dr. S. Lele of NASA Ames Research Center.

\section{REFERENCES}

1 Schlichting, H. Boundary-layer theory, $7^{\text {th }} \mathrm{ed}$., McGraw-Hill, New York, 1979, p. 93.

2 Davis, S. H. "The stability of time-periodic flows," Annual Review of Fluid Mechanics Vol. 8, 1976, p. 57.

3 Spalart, P. R. and Leonard, A. "Direct numerical simulation of equilibrium turbulent boundary layers," Turbulent Shear Flows 5 , 1986 , p. 234 (bound volume).

4 Spalart, P. R. "Numerical study of sink-flow boundary layers," Journal of Fluid Mechanics Vol. 172, 1986, p. 307.

$\underline{5}$ Townsend, A. A., The structure of turbulent shear flow, $2^{\text {nd }}$ ed., Cambridge University Press, Cambridge, 1976.

6 Spalart, P. R. "Numerical simulation of boundary layers: Part 1. Weak formulation and numerical method," NASA T. M. 88222, 1986.

I Hall, P. "The linear stability of flat Stokes layers," Royal Society London Vol. A 359, 1978, p. 151.

8 Monkewitz, P. A. and Bunster. A. "The stability of the Stokes layers: visual observations and some theoretical considerations," NASA/ICASE workshop "Stability of time-dependent and spatially-varying flows", Aug. 19-20, Hampton, VA. SpringerVerlag.

9 Merkli, P. and Thomann, H. "Transition to turbulence in oscillating pipe flow," Journal of Fluid Mechanics, Vol. 68, part 3, 1975, p. 567 .

10 Hino, M, Sawamoto, M. and Takasu, S. "Experiments on transition to turbulence in an oscillatory pipe flow," Journal of Fluid Mechanics, Vol. 75, part 2, 1976, p. 193.

11 Bradshaw, P., Cebeci, T. and Whitelaw, J. H. Engineering calculation methods for turbulent flow, Academic Press, New York, 1981.

12 Johnson, D. A. and King, L. S., "A mathematically simple turbulence closure model for attached and separated turbulent boundary layers," AIAA Journal, Vol. 23, No. 11., 1985, p. 1684. 


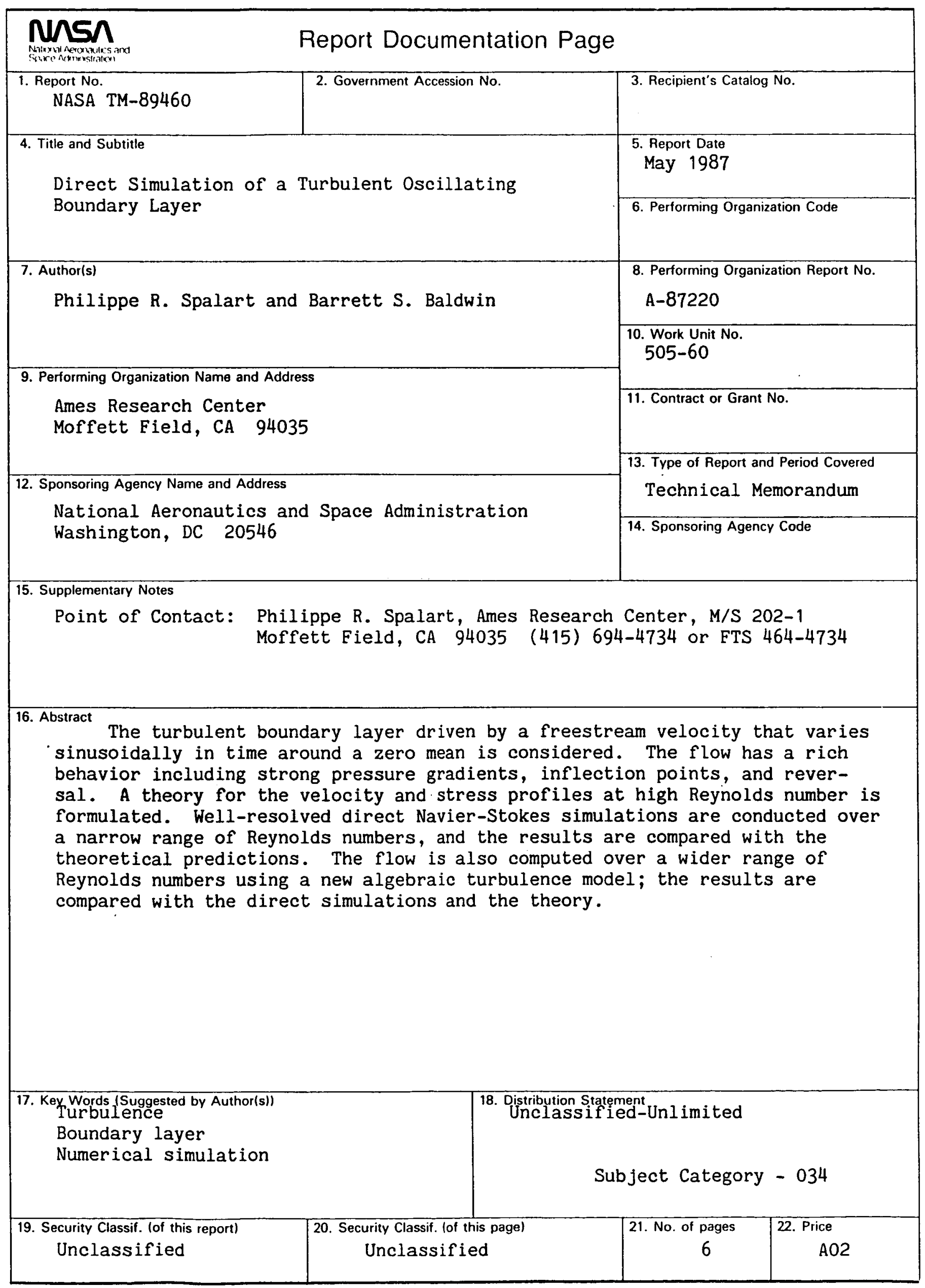

